University of Nebraska - Lincoln

DigitalCommons@University of Nebraska - Lincoln

Evgeny Tsymbal Publications

Research Papers in Physics and Astronomy

7-2006

\title{
Tunneling across a ferroelectric
}

Evgeny Y. Tsymbal

University of Nebraska at Lincoln, tsymbal@unl.edu

Hermann Kohlstedt

Institut für Festkörperforschung, Forschungszentrum Jülich, Jülich, Germany, hko@tf.uni-kiel.de

Follow this and additional works at: https://digitalcommons.unl.edu/physicstsymbal

Part of the Condensed Matter Physics Commons

Tsymbal, Evgeny Y. and Kohlstedt, Hermann, "Tunneling across a ferroelectric" (2006). Evgeny Tsymbal Publications. 22.

https://digitalcommons.unl.edu/physicstsymbal/22

This Article is brought to you for free and open access by the Research Papers in Physics and Astronomy at DigitalCommons@University of Nebraska - Lincoln. It has been accepted for inclusion in Evgeny Tsymbal Publications by an authorized administrator of DigitalCommons@University of Nebraska - Lincoln. 
Published in Science 313, issue: 5784 (July 14, 2006), pp. 181-183; doi 10.1126/science.1126230

Copyright (C) 2006 American Association for the Advancement of Science. Used by permission.

http://www.sciencemag.org

PERSPECTIVES • APPLIED PHYSICS

\title{
Tunneling across a Ferroelectric
}

\author{
Evgeny Y. Tsymbal ${ }^{1}$ and Hermann Kohlstedt ${ }^{2,3}$
}

${ }^{1}$ Department of Physics and Astronomy, Center for Materials Research and Analysis, University of Nebraska, Lincoln, Nebraska 68588, USA

2 Institut für Festkörperforschung, Forschungszentrum Jülich, D-52425 Jülich, Germany

${ }^{3}$ Department of Materials Science and Engineering, University of California, Berkeley, California 94720, USA

Spontaneously polarized materials through which electrons pass by tunneling may be used in novel electronic devices and may reveal new basic physics at the nanometer scale.

The phenomenon of electron tunneling has been known since the advent of quantum mechanics, but continues to enrich our understanding of many fields of physics, as well as creating sub-fields on its own. A tunnel junction consists of two metal electrodes separated by a nm-thick insulating barrier layer, as was first discussed by Frenkel in 1930 (1). Although classically forbidden, an electron can traverse the potential barrier that exceeds electron's energy, and hence has a non-vanishing probability to be found on the opposite side of the barrier. A famous example is electron tunneling in superconducting tunnel junctions discovered by Giaever that allowed measuring a quasiparticle energy gap in superconductors (2). More recently spindependent electron tunneling from ferromagnetic metal electrodes across an amorphous $\mathrm{Al}_{2} \mathrm{O}_{3}$ film was observed by Tedrow and Meservey (3). The latter discovery led Jullière to propose and demonstrate a magnetic tunnel junction in which the tunneling current depends on the relative magnetization orientation of the two ferromagnetic electrodes (4), the phenomenon nowadays known as tunneling (or junction) magnetoresistance (5).

A type of insulators which have been utilized as tunnel barriers is not limited to $\mathrm{Al}_{2} \mathrm{O}_{3}$. Several alternative barriers were successfully employed in tunnel junctions. For example, De Teresa et al. studied tunnel junctions with epitaxial perovskite $\mathrm{SrTiO}_{3}$ barriers to demonstrate the decisive role of interfaces in spin-dependent tunneling (6). Recently Parkin et al. (7) and Yuasa et al. (8) found large magnetoresistance in crystalline tunnel junctions with $\mathrm{MgO}$ barriers. Despite the diversity of materials used in tunnel junctions, the common feature of almost all the existing tunnel junctions is that they are based on non-polar barrier dielectrics.

Here we discuss the concept of a ferroelectric tunnel junction (FTJ) which takes the advantage of a ferroelectric as the barrier material. Ferroelectrics possess a spontaneous electric polarization that can be switched by an applied electric field. The discovery of ferroelectricity goes back to 1921 (9), i.e., approximately to the same time when the principles of quantum mechanical electron tunneling were formulated (1). The basic idea of a FTJ (called a polar switch at that time) belongs to Esaki et al. and was formulated in 1971 (10).

The concept of a FTJ is illustrated in Fig. 1, which shows a simplified band structure of a tunnel junction with a ferroelectric barrier. Due to a reversible electric polarization, FTJs are expected to have current-voltage characteristics different from those of conventional tunnel junctions. As was predicted by Esaki et al. (10), the electric-field-induced polarization reversal of a ferroelectric barrier may have a profound effect on the conductance of a FTJ, leading to 
resistive switching at the coercive field of the ferroelectric. Indeed, the polarization reversal alters sign of polarization charges at a barrier/electrode interface. Due to the incomplete screening this changes the depolarization field and hence the potential profile seen by transport electrons (11). It is interesting to note that the recent experimental (12) and theoretical (13) studies indicate that ionic displacements within the electrodes, in a few atomic monolayers adjacent to the ferroelectric, may affect the screening. The polarization switching alters positions of ions at the interfaces which influence the atomic orbital hybridizations at the interface and hence the transmission probability. Finally, due to piezoelectricity of a ferroelectric barrier an applied voltage produces a strain which changes transport characteristics of the barrier such as the barrier width and the attenuation constant (14). All these mechanisms are sketched in Fig.1.

A crucial condition to realize a FTJ is the existence of ferroelectricity in a nm-thick barrier film. Since ferroelectricity is a collective phenomenon thin films are expected to sustain a spontaneous electric polarization only above some critical thickness. It has been believed for a long time that the critical size for ferroelectricity is of a few tens nm. Such a property would render ferroelectrics to be useless for application as barriers in tunnel junctions. Triscone and his group in Geneva have, however, indicated that the critical size is much smaller than previously thought (15). They demonstrated the presence of a stable polarization in a 4-nm-thick epitaxial film of perovskite ferroelectric $\mathrm{Pb}\left(\mathrm{Zr}_{0.2} \mathrm{Ti}_{0.8}\right) \mathrm{O}_{3}$. The unambiguous experimental evidence for ferroelectricity in ultrathin epitaxial $\mathrm{PbTiO}_{3}$ films has recently arrived from the Argonne National Laboratory where structural investigations by synchrotron radiation demonstrated ferroelectric properties in these films down to $1.2 \mathrm{~nm}$ thickness (16). The modern theoretical studies based on density-functional theory by Ghosez and his group in Liège also support the existence of ferroelectricity in ultrathin perovskite films (17). In addition, ferroelectricity has been observed in mono molecular layers of ferroelectric polymers in form of polyvinylidene fluoride (PVDF) and its copolymers with trifluoroethylene (TrFE) (18). All these findings provide the undeniable experimental and theoretical evidence for viability of FTJs. As was recently pointed out by Dawber et al. (19), the idea of tunneling through a ferroelectric film has a considerable interest from the point of view of the fundamental science as well as device applications.

Functional properties of FTJs can be extended by replacing normal metal electrodes by ferromagnetic which makes the junctions multiferroic. The interplay between ferroelectric and ferromagnetic properties of the two ferroic constituents in a multiferroic tunnel junction (MFTJ) may affect the electric polarization of the ferroelectric barrier, the electronic and magnetic properties of the ferromagnet/ferroelectric interface and the spin polarization of the tunneling current. Such kinds of phenomena were observed by Ramesh and his group who discovered the coupling between ferroelectric and ferromagnetic order parameters in $\mathrm{BaTiO}_{3}-\mathrm{CoFe}_{2} \mathrm{O}_{4}$ nanostructures (20). These results indicate the potential to control the magnetization of the electrodes and consequently the spin-dependent electronic transport properties of MFTJs by electric fields. Another type of a MFTJ is feasible in which the barrier itself is made of a material that exhibits multiferroic properties in the bulk, such as $\mathrm{BiFeO}_{3}$ or $\mathrm{BiMnO}_{3}$. The research on bulk multiferroics that reveal simultaneously ferromagnetism and ferroelectricity started in Russia by Smolenskii et al. (21) in fifties of the last century. The interest in such materials was recently renewed due to the successful fabrication of multiferroic thin films as well as the deeper theoretical understanding of their properties (22). Recently Gajek et al. (23) showed that $\mathrm{BiMnO}_{3}$ tunnel barriers may serve as spin filters in magnetic tunnel junctions. This work was further advanced, to demonstrate the presence of ferroelectricity in ultrathin $\mathrm{BiMnO}_{3}$ films grown epitaxially on a half metallic $\mathrm{La}_{2 / 3} \mathrm{Sr}_{1 / 3} \mathrm{MnO}_{3}$ electrode (24). These studies open an avenue for the development of novel electronic devices in which a control of the ferroelectric polarization can 
be achieved by an external magnetic field via the magnetoelectric effect. Magnetoelectric properties of the barrier can also be used to produce an electrically-controlled exchange-bias magnetic field (25), thereby affecting the resistance of MFTJs (26).

In order to realize ferroelectric and multiferroic tunnel junctions a number of obstacles have to be overcome. In particular, parasitic effects such as local conductivity and transport via localized states have to be eliminated. In addition, the mechanisms of domain formation, nucleation and switching in nanoscale ferroelectrics and tunneling transport across polar thinfilm dielectrics have to be understood. Tremendous achievements in the field of complex oxide epitaxy and ultrathin ferroelectric polymers in addition to the nanoscale characterization techniques during the last years make a promise that all these problems can be solved and the realization of FTJs is just a matter of time. The diversity of interesting physical phenomena which control characteristics of these tunnel junctions and their multifunctional properties useful for various technological applications make the research in this field challenging and promising.

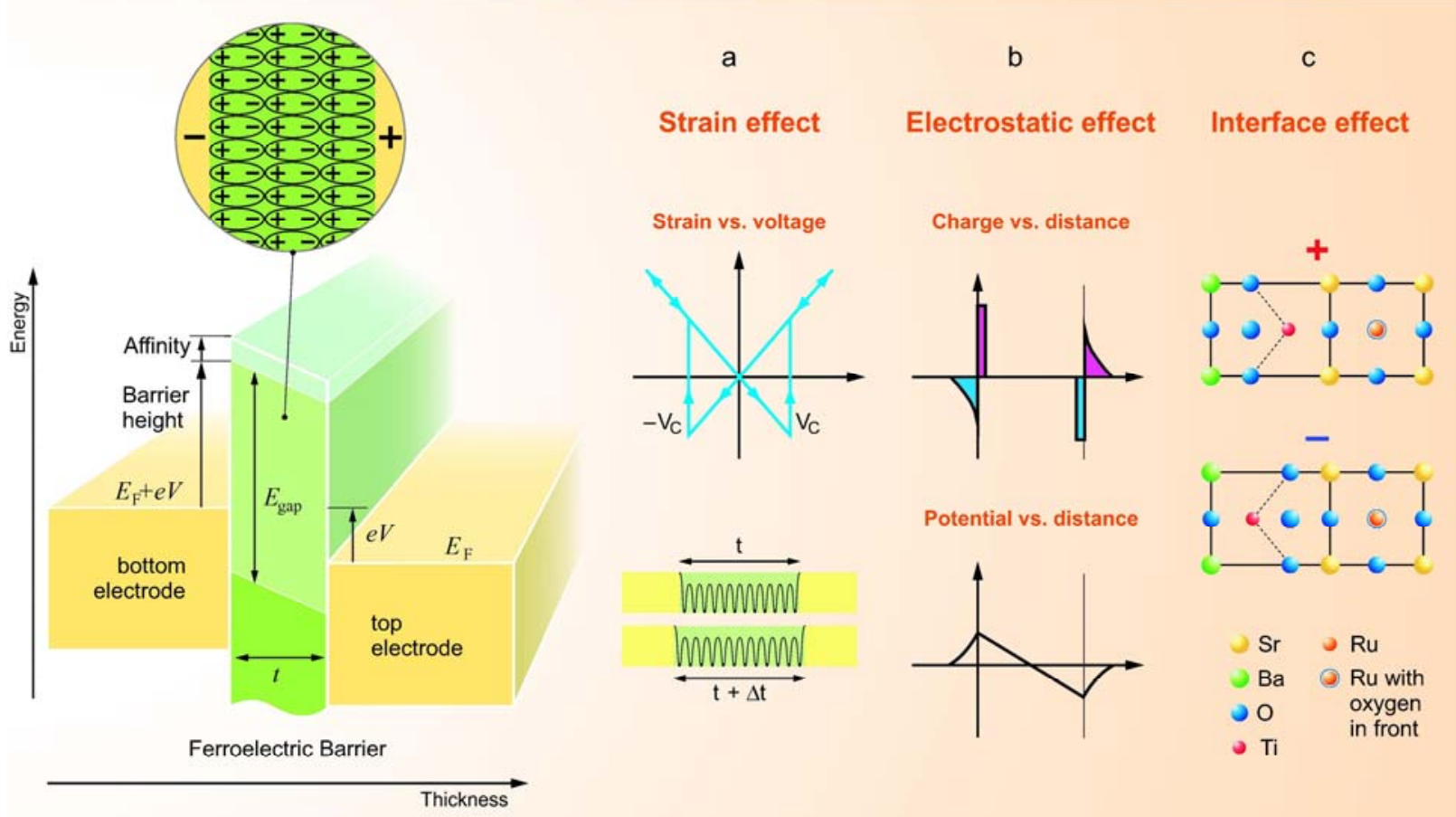

Fig.1 Schematic viewgraph of a ferroelectric tunnel junction which consists of two electrodes separated by a nm-thick ferroelectric barrier layer. The ferroelectric nature of the barrier changes the transmission probability in three ways:

a - Due to piezoelectricity of a ferroelectric barrier an applied voltage produces a strain which changes transport characteristics of the barrier such as the barrier width and the attenuation constant.

b - The incomplete screening of the ferroelectric bound charge leads to an electrostatic potential that superimposes the contact potential in the tunnel junction. 
c - The displacement of the Ti atom affects the atomic orbital hybridizations at the interface which makes the transmission probability different for the two opposite polarization orientations (here we consider a $\mathrm{BaTiO}_{3} / \mathrm{SrRuO}_{3}$ interface, as an example).

All three factors lead to resistive switching at the coercive voltage of the ferroelectric.

1. J. Frenkel, Phys. Rev. 36, 1604 (1930).

2. I. Giaever, Phys. Rev. Lett. 5, 147 (1960); ibid. 5, 464 (1960).

3. P. M. Tedrow, R. Meservey, Phys. Rev. Lett. 26,192 (1971); Phys. Rev. B 7, 318 (1973).

4. M. Jullière, Phys. Lett. A 54, 225 (1975).

5. J. S. Moodera et al., Phys. Rev. Lett. 74, 3273 (1995).

6. J. M. De Teresa et al., Science 286, 507 (1999).

7. S. S. P. Parkin et al., Nature Materials 3, 862 (2004).

8. S. Yuasa et al., Nature Materials 3, 868 (2004).

9. J. Valasek, Phys. Rev. 17, 475 (1921).

10. L. Esaki, R. B. Laibowitz, P. J. Stiles, IBM Tech. Discl. Bull. 13, 2161 (1971).

11. M. Ye. Zhuravlev et al., Phys. Rev. Lett. 94, 246802 (2005).

12 D. D. Fong et al., Phys. Rev B 71, 144112 (2005).

13 G. Gerra et al., Phys. Rev. Lett., in press.

14. H. Kohlstedt et al., Phys. Rev. B 72, 125341 (2005).

15. T. Tybell, C. H. Ahn, J.-M. Triscone, Appl. Phys. Lett. 75, 856 (1999).

16. D. D. Fong et al., Science 304, 1650 (2004).

17. J. Junquera, Ph. Ghosez, Nature 422, 506 (2003).

18. A. V. Bune et al., Nature 391, 874 (1998).

19. M. Dawber, K. M. Rabe, J. F. Scott, Rev. Mod. Phys. 77, 1083 (2005).

20. H. Zheng et al., Science 303, 661 (2004).

21. For a review, see G. A. Smolenskii and I. E. Chups, Sov. Phys. Usp. 25, 475 (1982).

22. N. A. Spaldin, M. Fiebig, Science 309, 391 (2005).

23. M. Gajek et al., Phys. Rev. B. 72, 020406 (2005).

24. H. Béa et al., Appl. Phys. Lett. 88, 062502 (2006).

25. P. Borisov et al., Phys. Rev. Lett. 94, 117203 (2005).

26. C. Binek, B. Doudin, J. Phys.: Condens. Matter 17, L39 (2005). 